\title{
ANÁLISE COMPARATIVA DOS CUSTOS DE DIFERENTES REDES DE DISTRIBUIÇÃO DE ENERGIA ELÉTRICA NO CONTEXTO DA ARBORIZAÇÃO URBANA ${ }^{1}$
}

\author{
Giuliana Del Nero Velasco ${ }^{2}$, Ana Maria Liner Pereira Lima ${ }^{3}$ e Hilton Thadeu Zarate do Couto ${ }^{4}$ \\ RESUMO - Com o intuito de estudar as podas feitas em árvores urbanas e analisar novas técnicas possíveis \\ de serem utilizadas para reduzir o número de podas e melhorar a convivência das espécies com a fiação elétrica, \\ além de permitir à árvore desempenhar plenamente suas funções ecológicas, buscou-se levantar dados referentes \\ aos três tipos principais de redes de distribuição de energia elétrica (aérea convencional, aérea compacta e \\ subterrânea), em quatro concessionárias de energia elétrica, a fim de compilá-los em um só trabalho. Foram \\ levantados custos de implantação das redes convencional, compacta e subterrânea, assim como custos de manutenção \\ e de poda de árvores. O custo de implantação de rede convencional (primária e secundária) variou de R \$54.188,39 \\ a R \$67.571,43. O custo de implantação da rede compacta (primária e secundária) foi em média R \$62.215,99. \\ Já o custo de implantação de uma rede subterrânea é, em média, de $\mathrm{R} \$ 436.585,04$. A rede compacta já é totalmente \\ viável, principalmente por ter custo de implantação praticamente igual à rede convencional e ter custo de \\ manutenção $80 \%$ menor. A rede subterrânea, embora com custo de implantação aproximadamente 10 vezes \\ maior quando comparado com o custo da convencional, por ter reduzido custo de manutenção e alta confiabilidade \\ do sistema elétrico, seu uso já pode ser viável em diversas ocasiões.
}

Palavras-chaves: Custos de redes de distribuição de energia elétrica e arborização viária.

\section{COSTS OF AERIAL ELECTRICITY CONVENTIONAL DISTRIBUTION NETWORKS, AERIAL COMPACT DISTRIBUTION NETWORKS AND UNDERGROUND NETWORKS IN ARBORICULTURE CONTEXT}

\begin{abstract}
In order to study the pruning carried out in urban trees and analyze new techniques that may be used to reduce pruning, improve the relationship between the species and the networks, and allow trees to fulfill their proper ecological functions, studies concerning the three main types of electric power distribution were carried out: aerial conventional distribution networks, aerial compact distribution networks and underground networks in four electric power companies. The study showed the implementation costs of the three named networks as well as pruning and tree maintenance costs. The costs of the conventional network (primary and secondary) ranged from $R \$ 54.188,39$ to $R \$ 67.571,43$. The costs for the compact network (primary and secondary) was on average $R \$ 62.215,99$, and the costs for the underground networks was on average $R \$$ 436.585,04. The compact network is now possible, mainly because its implementation costs are almost the same of the conventional network and its maintenance costs are $80 \%$ lower. Although the implementation costs of the underground network is 10 times higher than the conventional one, its lower maintenance costs and high reliability allow its use in several situations.
\end{abstract}

Keywords: Costs of electric power distribution system and street arborization.

\footnotetext{
${ }^{1}$ Recebido em 02.02.2005 e aceito para publicação em 05.04.2006.

${ }^{2}$ Programa de Pós-Graduação em Agronomia, Escola Superior de Agricultura “Luiz de Queiroz" - ESALQ/USP, Rua Borges de Barros, 175, apto 06, Vila Madalena, São Paulo-SP. E-mail: <gdnvelas @ esalq.usp.br>.

${ }^{3}$ Departamento de Produção Vegetal ESALQ/USP, Av. Pádua Dias, 11. Agronomia, Piracicaba-SP. E-mail: <amlplima@esalq.usp.br>.

${ }^{4}$ Departamento de Ciências Florestais ESALQ/USP, Av. Pádua Dias, 11. Agronomia, Piracicaba-SP. E-mail: <htzcouto@esalq.usp.br>.
} 


\section{INTRODUÇÃO}

A disputa entre as árvores nas calçadas e as redes elétricas pelo mesmo espaço é, sem dúvida, um dos principais problemas existentes na arborização viária de uma cidade, principalmente porque a tendência de plantar arbustos ou espécies de pequeno porte para que estes não interfiram na rede, assim como a poda dos galhos que já estão comprometidos com ela, ainda são as opções mais usadas pelos órgãos responsáveis. Segundo Soares (1998), são grandes as dificuldades de se implantar o verde nas cidades, principalmente conciliado à presença de equipamentos urbanos, como instalações hidráulicas e redes elétricas, telefônicas ou sanitárias. Além disso, não se pode afirmar que a poda soluciona o problema da convivência entre árvore e fiação elétrica, visto que, segundo Browning (1997), a maioria das árvores, uma vez podadas, começa a brotar em direção aos condutores elétricos e precisa de novas manutenções em curto espaço de tempo.

Segundo Seitz (1990), a poda é uma agressão a um organismo vivo, com estruturas e funções definidas e alguns mecanismos de defesa contra seus inimigos naturais. A prática da poda não deve ser totalmente abolida, mas sim usada de forma correta nas árvores urbanas, evitando que grandes erros sejam cometidos na ilusão de estar realizando o melhor para as plantas.

São três os tipos principais de redes de distribuição de energia elétrica abordados no presente trabalho: a rede de distribuição aérea convencional, a aérea compacta e a subterrânea.

Segundo Sardeto (1999), a rede convencional é caracterizada por condutores nus, apoiados sobre isoladores de vidro ou porcelana, fixados horizontalmente sobre cruzetas de madeira, nos circuitos de média tensão e, verticalmente, nos de baixa tensão. Essa rede fica totalmente desprotegida contra as influências do meio ambiente, apresenta alta taxa de falhas e exige que sejam feitas podas drásticas nas árvores, visto que o simples contato do condutor nu com um galho de árvore pode provocar o desligamento de parte da rede.

Já a rede compacta ou rede protegida é um sistema de distribuição de energia elétrica aéreo, no qual a rede primária é constituída de três condutores cobertos por uma camada de polietileno reticulado - XLPE (composto extrudado de polietileno termofixo), sustentados por um cabo mensageiro de aço $(\operatorname{com} 9,5$ mm de diâmetro, de alta resistência), o qual, por sua vez, sustenta espaçadores plásticos (polietileno de alta densidade - HDPE, dotados de anéis ou laços poliméricos, para amarração dos condutores e do mensageiro). Esses espaçadores são instalados em intervalos de 8 a $10 \mathrm{~m}$, apoiando os condutores que ficam dispostos em um arranjo triangular compacto. Cabe ressaltar que os cabos protegidos são apenas "encapados", não podendo ser considerados como "isolados eletricamente", por não terem seu campo elétrico confinado. O cabo mensageiro de sustentação é, por sua vez, fixado aos postes, através de uma ferragem metálica chamada de braço suporte tipo "L” (SARDETO, 1999).

Por fim, o sistema subterrâneo de distribuição de energia elétrica, sem dúvida, é mais complexo que o sistema aéreo, além de um custo mais elevado, mas também, em contrapartida, segundo Boccuzzi et al. (1997), apresenta uma série de benefícios, como:

a) Redução significativa das interrupções pela diminuição da exposição dos circuitos aos agentes externos, incrementando, assim, a confiabilidade do serviço.

b) Eliminação dos circuitos aéreos, o que melhora bastante a aparência do sistema e, principalmente, ajuda a preservar as árvores, contribuindo, conseqüentemente, para o embelezamento das cidades e conservação do meio ambiente.

c) Aumento da segurança para a população, com a redução do risco de acidentes por ruptura de condutores e contatos acidentais.

d) Redução dos custos de manutenção, como podas de árvores e deslocamento de turmas de emergência.

O objetivo do presente trabalho foi comparar e analisar dados referentes a custos de redes de distribuição de energia elétrica e de manutenção de árvores, bem como custos de podas, com o propósito de desmistificar a impossibilidade de se utilizarem novas redes de distribuição (compacta e subterrânea) como alternativa para as redes aéreas convencionais. Busca-se, com isso, defender a substituição de redes aéreas convencionais por redes compactas ou subterrâneas, visando propiciar às árvores a realização - de fato de sua função no meio urbano. 


\section{MATERIAL E MÉTODOS}

Foram levantados custos de implantação, manutenção e substituição, referentes às redes de distribuição de energia elétrica convencional, compacta e subterrânea, nas concessionárias de energia elétrica, CPFL/SP, da cidade de Campinas, SP, COPEL/PR, da cidade de Maringá, PR, CEMIG/MG, da cidade de Belo Horizonte, MG e LIGHT/ RJ, da cidade do Rio de Janeiro, RJ.

Visto os dados terem sido obtidos em diferentes anos, para que pudessem ser comparados foi feito um deflacionamento com base no índice IPC - geral - RMSP (Índice de Preços ao Consumidor-Região Metropolitana de São Paulo), utilizando-se o IPC geral de dezembro de 1999, que foi de 179,2904, e o de dezembro de 1998, no valor de 165,0468 .

Os custos foram apresentados em $\mathrm{R} \$ / \mathrm{km}$. Alguns valores foram obtidos em $\mathrm{R} \$ /$ poste, sendo que, considerando a existência de um poste a cada $35 \mathrm{~m}$, ou seja, 30 postes em $1 \mathrm{~km}$, o valor obtido foi multiplicado por 30, obtendo-se um valor final em $\mathrm{R} \$ / \mathrm{km}$.

Para o cálculo da parcela referente à poda, inclusa no total do custo de manutenção preventiva, pela CEMIG/ MG, foi utilizada a seguinte fórmula, com base em CEMIG (1998):

$\mathrm{PA}=\mathrm{Pe}\{(\mathrm{LM}$ x árvore/vão x Spa $)+(\mathrm{LV} x$ árvore/vão x Spa) $\}$,

em que:
PA = custo de poda por árvore;

$\mathrm{Pe}=$ periodicidade de poda;

$\mathrm{LM}=$ porcentagem de serviços executados com turmas de linha morta;

Árvore/vão $=3$, representando quantas árvores existem em um vão de rede urbana, isto é, na distância entre dois postes (de concreto, redondos ou quadrados), fincados na calçada;

$\mathrm{Spa}=$ serviço pago para podar uma árvore; e

$\mathrm{LV}=$ porcentagem de serviços executados com turmas de linha viva.

\section{RESULTADOS E DISCUSSÃO}

\subsection{Levantamento dos custos}

Houve grande variação nos custos das redes, assim como na sua caracterização e nomenclatura, variando muito de região para região. Dessa forma, o presente trabalho procurou obter o máximo de informações de diferentes regiões para mostrar a variação entre elas.

\subsubsection{Rede de distribuição aérea convencional}

ATabela 1 reúne os custos obtidos nas três concessionárias deenergiaelétrica(CPFL/SP,COPEL/PReCEMIG/MG). Notase que os valores fornecidos pela COPEL e pela CEMIG foram corrigidos de 1999 para 2001 e de 1998 para 2001, respectivamente, para que pudessem ser comparados aos custos fornecidos pela CPFL.

Tabela 1 - Custos (médio e corrigido) para implantação de rede aérea convencional nas concessionárias CPFL/SP, COPEL/ PR e CEMIG/MG

Table 1 - Costs (average and corrected) to implement aerial conventional network for CPFL/SP, COPEL/PR and CEMIG/MG

\begin{tabular}{|c|c|c|c|c|}
\hline & $\begin{array}{c}\text { Tipo de Rede Aérea } \\
\text { Convencional } \\
\end{array}$ & $\begin{array}{l}\text { Custo em } \\
\mathrm{R} \$ / \text { Poste }\end{array}$ & $\begin{array}{c}\text { Custo em } \\
\mathrm{R} \$ / \mathrm{km}\end{array}$ & $\begin{array}{l}\text { Custo Corrigido em } \\
\mathrm{R} \$ / \mathrm{km}(\mathrm{em} \mathrm{2001)}\end{array}$ \\
\hline \multirow[t]{3}{*}{$\mathrm{CPFL} / \mathrm{SP}^{5}$} & Rede primária & - & $39.000,00(\mathrm{em} \mathrm{2001)}$ & - \\
\hline & Rede secundária & - & $28.571,43$ (em 2001) & - \\
\hline & $\begin{array}{c}\text { Rede primária }+ \\
\text { secundária }\end{array}$ & - & $67.571,43$ (em 2001) & - \\
\hline \multirow[t]{2}{*}{$\overline{\text { COPEL} / \mathrm{PR}^{6}}$} & $\begin{array}{c}\text { Rede primária com } \\
\text { bitola } 336,4 \text { MCM AWG }\end{array}$ & - & $17.910,03$ (em 1999) & $20.028,83$ \\
\hline & $\begin{array}{l}\text { Rede primária com } \\
\text { bitola } 2 \mathrm{AWG}\end{array}$ & - & $10.465,07$ (em 1999) & $11.703,11$ \\
\hline$\overline{\mathrm{CEMIG} / \mathrm{MG}^{7}}$ & $\begin{array}{c}\text { Rede primária } \\
+ \text { secundária }\end{array}$ & $1.486,88$ & $44.606,40$ (em 1998) & $54.188,39$ \\
\hline
\end{tabular}

${ }^{5}$ CAVAlCANTE, J. A. (Engenheiro - CPFL - Companhia Paulista de Força e Luz, Campinas - São Paulo). Comunicação pessoal, 2002.

${ }^{6}$ Fonte: Sardeto, 1999.

${ }^{7}$ Fonte: adaptado de CEMIG, 1998. 
Os valores da Tabela 1 confirmam que os dados obtidos das concessionárias são bem distintos entre si, havendo grande variação dos valores de custos, provavelmente por se tratar de locais diferentes e distantes, em estados diferentes, além do fato de que as concessionárias utilizam material variado para a construção de suas redes, acarretando em custos também distintos. Além disso, é importante ressaltar que, tanto a CPFL/SP quanto a CEMIG/MG, refere-se a custos de redes primária + secundária, ao passo que a COPEL/ $\mathrm{PR}$ se refere a custo apenas de rede primária de distribuição de energia elétrica.

\subsubsection{Rede de distribuição aérea compacta (rede protegida)}

Baseado em dados da COPEL/PR e CEMIG/MG, têm-se na Tabela 2 os custos de implantação da rede de distribuição compacta.

Assim como para rede convencional, cabe ressaltar que a CEMIG/MG, ao contrário da COPEL/PR, referese ao custo de rede primária + secundária, nesse caso rede primária compacta e secundária isolada.

\subsubsection{Rede de distribuição subterrânea}

Segundo dados da LIGHT $^{10}$, o custo total de 1 km de rede de distribuição subterrânea envolve os custos de abertura de valas, recapeamento (obras civis) e todos os equipamentos utilizados (cabos, transformadores e emendas). O custo varia de acordo com os diversos tipos de cabos possíveis de serem utilizados. Dessa forma, na Tabela 3 apresenta-se o cálculo do custo para $1 \mathrm{~km}$ de rede subterrânea.
Segundo Bernis ${ }^{1}{ }^{1}$, as manutenções em redes subterrâneas são raríssimas e esporádicas (falha em cabo ou em conexão e desgaste de chaves, entre outros). No entanto, os reparos são mais elaborados e especializados e, com isso, requerem mais tempo de mão-de-obra. Mas esse custo é diluído ao longo do tempo, pois os intervalos entre esses reparos são muito longos em função da alta confiabilidade da rede. Assim, pode-se dizer que a redução das manutenções seria superior a duas vezes ao despendido em uma rede aérea (que requer manutenções periódicas).

Sabe-se que as redes de distribuição subterrânea já estão sendo utilizadas em alguns condomínios fechados de alto padrão, podendo-se citar alguns exemplos: de acordo com Pirelli (2000), em um condomínio cujas residências foram avaliadas em US\$ 45 mil, o custo da rede subterrânea por casa foi de US\$ 561. Se fosse adotada a rede aérea seria, US\$ 134. Assim, a diferença ao se adotar uma rede subterrânea é de US\$ 427, ou seja, apenas $0,95 \%$ do imóvel. Outro exemplo cita um condomínio de casas avaliadas individualmente em US\$ 85 mil, no qual o custo da rede subterrânea por casa foi de US\$ 965. Para rede aérea seria de US\$ 199, uma diferença de US\$ 766, representando apenas $0,90 \%$ do valor do imóvel. Dessa forma, esses dados indicam que a instalação de redes subterrâneas representa um investimento inferior a $1 \%$ do custo do imóvel, sendo que, "considerando os ganhos econômicos, estéticos e de segurança, fica claro que não vale mais a pena instalar redes aéreas, pois a valorização final do imóvel será bem maior com redes subterrâneas".

Tabela 2 - Custos (médio e corrigido) para implantação de rede aérea compacta, nas concessionárias COPEL/PR e CEMIG/ MG

Table 2 - Cost (average and corrected) to implant the aerial compact net at COPEL/PR and CEMIG/MG

\begin{tabular}{|c|c|c|c|c|}
\hline & $\begin{array}{c}\text { Tipo de Rede Aérea } \\
\text { Compacta }\end{array}$ & $\begin{array}{l}\text { Custo em } \\
\mathrm{R} \$ / \text { Poste }\end{array}$ & $\begin{array}{c}\text { Custo em } \\
\mathrm{R} \$ / \mathrm{km}\end{array}$ & $\begin{array}{l}\text { Custo Corrigido em } \\
\mathrm{R} \$ / \mathrm{km}(\mathrm{em} \mathrm{2001)}\end{array}$ \\
\hline \multirow[t]{2}{*}{ COPEL/PR ${ }^{8}$} & $\begin{array}{l}\text { Rede primária com } \\
\text { bitola } 336,4 \text { MCM XLPE }\end{array}$ & - & $45.175,27$ (em 1999) & $50.519,61$ \\
\hline & $\begin{array}{c}\text { Rede primária com } \\
\text { bitola } 2 \text { XLPE }\end{array}$ & - & $32.802,81$ (em 1999) & $36.683,46$ \\
\hline CEMIG/MG ${ }^{9}$ & Rede primária + secundária & $1.707,15$ & $51.214,50(\mathrm{em} \mathrm{1998)}$ & $62.215,99$ \\
\hline
\end{tabular}

${ }^{8}$ Fonte: adaptado de Sardeto, 1999

${ }^{9}$ Fonte: adaptado de CEMIG, 1998.

${ }^{10}$ LIGHT. Rio de Janeiro - RJ, Comunicação Pessoal, 2003.

${ }^{11}$ BERNIS, R. A. O. (Engenheiro - CEMIG - Companhia Energética de Minas Gerais, Belo Horizonte - Minas Gerais). Comunicação pessoal, 2002.

R. Árvore, Viçosa-MG, v.30, n.4, p.679-686, 2006 
Tabela 3 - Custos parciais e custo total de $1 \mathrm{~km}$ de rede de distribuição subterrânea, LIGHT/RJ, em 2001 Table 3 - Partial and total costs for $1 \mathrm{~km}$ of underground network, LIGHT/RJ in 2001

\begin{tabular}{llcr}
\hline Equipamentos Utilizados & \multicolumn{1}{c}{ Preço } & $\begin{array}{c}\text { Quantidade } \\
\text { Necessária }\end{array}$ & $\begin{array}{c}\text { Custo Parcial } \\
(\mathrm{R} \$ / \mathrm{km})\end{array}$ \\
\hline $\begin{array}{l}\text { Cabo } 240 \mathrm{~mm}^{2} \text { de cobre } \\
\text { (rede de média tensão) }\end{array}$ & $\mathrm{R} \$ 74,93 /$ metro & 1000 metros & $74.933,95$ \\
$\begin{array}{l}\text { Cabo } 240 \mathrm{~mm}^{2} \text { de alumínio armado } \\
\text { (rede de baixa tensão) }\end{array}$ & $\mathrm{R} \$ 49,63 /$ metro & 50 metros & 2481,54 \\
$\begin{array}{l}\text { Terminais de alta tensão } \\
\left.\text { (para cabo 95 } \mathrm{mm}^{2} \mathrm{Al}\right)\end{array}$ & $\mathrm{R} \$ 374,89 /$ conjunto & 2 conjuntos & 749,79 \\
$\begin{array}{l}\text { Transformador pedestal 300 kVA } \\
\text { Via asfaltada com recalçamento em }\end{array}$ & $\mathrm{R} \$ 15445,81 /$ conjunto & 1 conjunto & 15445,81 \\
$\begin{array}{l}\text { asfalto (obra civil) } \\
\begin{array}{l}\text { Vala em via asfaltada com } \\
\text { recalçamento em asfalto (obra civil) }\end{array}\end{array}$ & $\mathrm{R} \$ 174,55 /$ metro $\$ 168,43 /$ metro & 1.000 metros & $174.548,08$ \\
\hline CUSTO TOTAL & & 1.000 metros & $168.425,88$ \\
\hline
\end{tabular}

\subsubsection{Transformação da rede convencional para a compacta protegida}

Para a obtenção do custo de substituição de rede convencional por rede compacta, considerouse o reaproveitamento do material da rede nua existente.

Isso é possível, visto que para a transformação da rede convencional para a compacta pode-se utilizar o mesmo traçado e quase a totalidade dos postes já em uso, praticamente não existindo necessidades de obras civis. $O$ trabalho de desmontagem da rede antiga e montagem da nova é relativamente simples e rápido, além de poder ser feito em etapas programadas (COPEL, 1995).

Dessa forma, os custos de transformação fornecidos pela COPEL/PR e CPFL/SP são mostrados na Tabela 4

Como pode ser observado em ambas as concessionárias de energia elétrica, a transformação é mais barata que a implantação de novas redes convencionais, o que por si só já justificaria investimentos, talvez até subsidiados por algumas firmas interessadas em propagandas, principalmente visando a preservação de árvores antigas e, ou, frondosas, em áreas onde o conflito é inevitável.

\subsubsection{Custos de manutenção (custos operacionais)}

Segundo a CEMIG (1998), são dois os tipos de manutenção de redes de distribuição: manutenção preventiva e manutenção corretiva.

Tabela 4 - Custos (médio e corrigido) para transformação de rede convencional em rede compacta, nas concessionárias COPEL/PR e CPFL/SP

Table 4 - Costs (average and corrected) to change the conventional network into compact network, for COPEL/PR and CPFL/SP

\begin{tabular}{|c|c|c|c|}
\hline & Tipo de rede & $\begin{array}{l}\text { Custo em } \\
\mathrm{R} \$ / \mathrm{km}\end{array}$ & $\begin{array}{l}\text { Custo corrigido em } \\
\mathrm{R} \$ / \mathrm{km}(\mathrm{em} \mathrm{2001)} \\
\end{array}$ \\
\hline \multirow[t]{2}{*}{ COPEL/PR ${ }^{12}$} & $\begin{array}{l}\text { Rede primária compacta com } \\
\text { bitola } 336,4 \text { MCM XLPE }\end{array}$ & $38.408,03$ (em 1999) & $42.951,79$ \\
\hline & $\begin{array}{c}\text { Rede primária compacta } \\
\text { com bitola } 2 \text { XLPE }\end{array}$ & $27.777,50$ (em 1999) & $31.063,64$ \\
\hline $\mathrm{CPFL} / \mathrm{SP}^{13}$ & $\begin{array}{c}\text { Rede compacta } \\
\text { (primária }+ \text { secundária) }\end{array}$ & $35.000,00($ em 2001) & - \\
\hline
\end{tabular}

R. Árvore, Viçosa-MG, v.30, n.4, p.679-686, 2006 


\subsubsection{Manutenção preventiva}

Este tipo de manutenção refere-se à manutenção geral das estruturas, condutores e equipamentos, especialmente na verificação de pontos quentes em conexões, chaves e fuga de corrente em isoladores, entre outros. Além desses pontos, a manutenção preventiva engloba os gastos com a poda das árvores e a retirada de objetos da rede.

A Tabela 5 reúne os custos de manutenção preventiva, tanto da CEMIG/MG quanto da CPFL/ SP, segundo Cavalcante.

Percebe-se que os valores fornecidos pelas concessionárias diferem muito entre si, em razão, provavelmente, dos critérios adotados para cálculo desses custos.

\subsubsection{Manutenção corretiva}

De acordo com CEMIG (1998), esse tipo de manutenção dá-se em função do número de interrupções acidentais e do tempo do restabelecimento da interrupção urbana. Durante essa manutenção, ocorrem as substituições de material danificado, além das manobras necessárias para a execução dos serviços na rede.

A Tabela 6 reúne os custos de manutenção corretiva, tanto para a CEMIG/MG (adaptado de CEMIG, 1998), quanto para a CPFL/SP.

Nota-se que, se por um lado, a rede aérea convencional (RDA) tem menor custo de instalação quando comparada com a compacta, por outro ela tem um custo de manutenção no mínimo duas vezes maior após a instalação.

\subsubsection{Manutenção preventiva x poda}

Como já comentado, o custo da poda está incluso dentro do custo de manutenção preventiva, sendo seus valores apresentados na Tabela 7 .

Nota-se, na Tabela 7, que os valores de poda nas redes RSI e RDP não são muito distintos. Isso se deve ao fato de que o circuito secundário é o mais baixo da rede e, portanto, é o que afeta primeiro as árvores. Assim, tanto na RSI quanto na RDP a rede de baixa tensão é isolada, não havendo muita diferença em relação à interferência com a arborização.

A redução maior se dá em comparação entre a RDA e a RDP, em que a redução do custo de poda é da ordem de cinco vezes (de $R \$ 68,82$ para $R \$ 14,12$ ), ou seja, uma redução de $79,5 \%$ no custo de poda.

Já para Cavalcante, na CPFL, o custo de poda varia de acordo com a altura da árvore, se já atingiu a rede primária ou se está apenas interferindo na rede secundária. Isso porque na rede secundária a poda pode ser feita com turmas de linha morta, ou seja, com a rede desligada; já na rede primária normalmente a poda é feita com turmas de linha viva, com a rede ligada. Os custos de poda, tanto em rede secundária quanto em primária, estão na Tabela 8 .

Ainda, segundo informações da CPFL, cada árvore é podada uma vez ao ano, independente de a fiação ser compacta ou convencional. O custo da poda também não varia de acordo com o tipo de fiação; apenas o tipo de poda pode ser considerado diferente quando se comparam rede aérea convencional e compacta protegida.

\subsubsection{Visão geral dos custos}

Foi feita uma tabela com os principais custos já citados no presente trabalho, para que se possa ter uma visão geral, facilitando sua comparação. Notouse a dificuldade em padronizar os dados, visto cada concessionária fornecê-los de maneira diferente ( $\mathrm{R} \$$ /

Tabela 5 - Custos (em 1998) e custos corrigidos (em 2001) de manutenção preventiva na rede aérea convencional (RDA), secundária isolada (RSI) e compacta (RDP), com e sem a presença de arborização, CEMIG/MG e custo de manutenção preventiva na rede aérea convencional, no ano de 2001, CPFL/SP

Table 5 - Costs (in 1998) and corrected (in 2001) for Preventive Maintenance of aerial conventional network, secondary isolated e compact, with and without trees, CEMIG/MG and Costs of preventive maintenance of aerial conventional network, 2001, CPFL/SP

\begin{tabular}{ccccccc}
\hline Manutenção Preventiva & \multicolumn{2}{c}{ RDA } & \multicolumn{2}{c}{ RSI } & RDP \\
\hline R \$/Km & Com & Sem & Com & Sem & Com \\
& arborização & arborização & arborização & arborização & $\begin{array}{c}\text { Sem } \\
\text { arborização }\end{array}$ & arborização \\
\hline CEMIG (1998) & 108,00 & 51,43 & 43,57 & 29,52 & 17,08 \\
CEMIG (2001) & 131,20 & 62,48 & 52,93 & 35,86 & 20,75 & $-5,51$ \\
CPFL (2001) & 760,00 & - & - & - & - \\
\hline
\end{tabular}

R. Árvore, Viçosa-MG, v.30, n.4, p.679-686, 2006 
km; R \$poste; custo referente à rede primária; e custo referente a redes primária e secundária, entre outros). Esses valores reunidos podem ser vistos na Tabela 9.

Tabela 6 - Custos (em 1998) e custos corrigidos (em 2001) de Manutenção Corretiva na rede aérea convencional (RDA), secundária isolada (RSI) e compacta (RDP), CEMIG/MG e custo de Manutenção Corretiva na rede aérea convencional, CPFL/SP, no ano de 2001

Table 6 - Costs (in 1998) and corrected costs (in 2001) for corrective maintenance of the aerial conventional network, secondary isolated and compact, CEMIG/ $M G$, and costs of corrective maintenance for aerial conventional network, $C P F L / S P, 2001$

\begin{tabular}{cccc}
\hline Manutenção Corretiva (R $\$ / K m)$ & RDA & RSI & RDP \\
\hline CEMIG (1998) & 15,41 & 7,48 & 3,19 \\
CEMIG (2001) & 18,72 & 9,09 & 3,88 \\
CPFL (2001) & $1.600,00$ & & \\
\hline
\end{tabular}

Tabela 7 - Custo de poda nas três redes de distribuição aérea: convencional (RDA), secundária isolada (RSI) e compacta (RDP), CEMIG/MG, em 1 ano

Table 7-Prunning costs of the three aerial distribution networks: conventional, secondary isolated and compact, CEMIG/ $M G$ for a year

\begin{tabular}{cccc}
\hline & $\begin{array}{c}\text { Tipo } \\
\text { de rede }\end{array}$ & $\begin{array}{c}\text { Custo Total Anual } \\
\text { da Manutenção Preventiva } \\
\text { em Locais com Arborização }\end{array}$ & $\begin{array}{c}\text { Parcela } \\
\text { Referente } \\
\text { à Poda }\end{array}$ \\
\hline RDA & 1998 & $\mathrm{R} \$ 108,00$ & $\mathrm{R} \$ 56,65$ \\
& 2001 & $\mathrm{R} \$ 131,20$ & $\mathrm{R} \$ 68,82$ \\
$\mathrm{RSI}$ & 1998 & $\mathrm{R} \$ 43,57$ & $\mathrm{R} \$ 14,02$ \\
& 2001 & $\mathrm{R} \$ 52,93$ & $\mathrm{R} \$ 17,03$ \\
$\mathrm{RDP}$ & 1998 & $\mathrm{R} \$ 17,08$ & $\mathrm{R} \$ 11,62$ \\
& 2001 & $\mathrm{R} \$ 20,75$ & $\mathrm{R} \$ 14,12$ \\
\hline
\end{tabular}

É essencial ressaltar que, do total do custo de implantação de rede subterrânea ( $\mathrm{R} \$ 436.585,04 / \mathrm{km})$, $\mathrm{R} \$ 342.973,96 / \mathrm{km}$ referem-se a obras civis, o que representa $78,5 \%$ do custo total da rede. Se, como exemplo, fosse tirado o valor das obras civis, o custo da rede cairia para $\mathrm{R} \$ 93.611,08 / \mathrm{km}$, totalmente acessível quando comparado a um custo de $\mathrm{R} \$ 67.571,43 / \mathrm{km}$ de rede convencional. As obras civis poderão em breve ter seu custo reduzido, visto que já existem cabos com boa resistência que podem ser diretamente enterrados no solo, evitando a abertura de valas e obras.

Dessa forma, acredita-se que o sistema subterrâneo, em breve, será totalmente viável e seu preço sofrerá uma redução significativa, visto que novas tecnologias e alternativas em relação à implantação dessas redes já estão sendo testadas e estudadas. Como exemplo disso, pode-se afirmar que já está em uso o sistema de distribuição subterrânea residencial, o qual é bem mais barato que o subterrâneo propriamente dito, pois, de acordo com Almeida et al. (2000), são utilizados dutos fabricados em Polietileno de Alta Densidade (PEAD), em substituição aos de cimento-amianto, os quais podem ser instalados diretamente no solo, dispensando as caixas de concreto.

Tabela 8 - Custos de poda por árvore em redes primárias e secundárias de distribuição de energia elétrica, CPFL/SP, em 2001

Table 8 - Prunning costs per tree in primary and secondary networks

\begin{tabular}{lc}
\hline Tipo de Rede Aérea & Custo de Poda/Árvore (r\$) \\
\hline Primária - classe $15 \mathrm{kV}$ & 20,00 \\
Secundária $-220 / 127 \mathrm{~V}$ & 6,00 \\
\hline
\end{tabular}

Tabela 9 - Custos referentes a redes convencionais, compactas e subterrâneas, em reais/km e custos de podas, em reais/ árvore, nas concessionárias CPFL/SP, COPEL/PR, CEMIG/MG e LIGHT/RJ

Table 9 - Costs referring to conventional, compact and underground networks, in reais/Km plus costs of tree pruning, in Reais/tree, of the following electric power companies: CPFL/SP, COPEL/PR, CEMIG/MG and LIGHT/RJ

\begin{tabular}{|c|c|c|c|c|}
\hline & CPFL/SP & COPEL/PR & CEMIG/MG & $\mathrm{LIGHT/RJ}$ \\
\hline Implantação de rede convencional & $\begin{array}{c}67.571,43 \\
\left(1^{\text {aria }}+2^{\text {aria }}\right)\end{array}$ & $\begin{array}{l}\text { De } 11.703,83 \\
\text { à } 20.028,83\left(1^{\text {aria }}\right)\end{array}$ & $\begin{array}{c}54.188,39 \\
\left(1^{\text {aria }}+2^{\text {aria }}\right)\end{array}$ & - \\
\hline Implantação de rede compacta & - & $\begin{array}{c}\text { De } 36.519,61\left(1^{\text {aria }}\right) \\
\text { à } 50.519,61\end{array}$ & $\begin{array}{c}62.215,99 \\
\left(1^{\text {aria }}+2^{\text {aria }}\right)\end{array}$ & - \\
\hline Implantação de rede subterrânea & - & - & - & $436.585,04$ \\
\hline $\begin{array}{l}\text { Transformação de rede } \\
\text { convencional para compacta }\end{array}$ & $35.000,00$ & $\begin{array}{l}\text { De } 31.063,64 \\
\text { à } 42.951,79\end{array}$ & - & - \\
\hline Manutenção preventiva de rede convencional & 760,00 & - & 131,20 & - \\
\hline Manutenção preventiva de rede compacta & - & - & 20,75 & - \\
\hline Manutenção corretiva de rede convencional & $1.600,00$ & - & 18,72 & - \\
\hline Manutenção corretiva de rede compacta & - & - & 3,88 & - \\
\hline Poda em rede convencional & $\begin{array}{c}20,00\left(1^{\text {aria }}\right) \\
6,00\left(2^{\text {aria }}\right)\end{array}$ & - & $\begin{array}{c}68,82 \\
\left(1^{\text {aria }}+2^{\text {aria }}\right)\end{array}$ & $\begin{array}{c}32,02 \\
\left(1^{\text {aria }}+2^{\text {aria }}\right)\end{array}$ \\
\hline Poda em rede compacta & - & - & 14,12 & - \\
\hline
\end{tabular}


Além dos dutos, também são dispensadas as câmaras subterrâneas, que ocupam muito espaço no subsolo e tem custo elevado, sendo os transformadores isolados colocados em cubículos metálicos e montados sobre as calçadas (caixas), o que reduz o custo atual de uma câmara subterrânea de $\mathrm{R} \$ 8.000,00$ para $\mathrm{R} \$ 800,00$, preço das novas caixas propostas.

\section{CONCLUSÃO}

As principais conclusões do presente trabalho foram:

* Custo de implantação da rede compacta é praticamente o mesmo da rede convencional.

* Há uma redução de $80 \%$ no custo de manutenção da rede compacta quando comparado à rede convencional.

* É mais vantajoso o uso de redes de distribuição compacta em vez de redes convencionais, tanto em termos de custos quanto de benefícios entre esses a melhor convivência com as árvores viárias.

* Custo de implantação de rede subterrânea é 10 vezes maior e de manutenção duas vezes menor quando comparado com a rede convencional.

* Uso de rede subterrânea já é viável em diversas ocasiões, devendo cada caso ser estudado e ter sua viabilidade analisada, visto que, em muitos deles, tornase vantajoso fazer um investimento mais alto, para depois contar com uma manutenção mínima, de alta confiabilidade do sistema, sem riscos para as populações humanas e vegetais da área.

\section{AGRADECIMENTOS}

Às Companhias de Energia Elétrica, COPEL/PR, CPFL/SP, CEMIG/MG e LIGHT/RJ, pelas informações concedidas.

\section{REFERÊNCIAS BIBLIOGRÁFICAS}

ALMEIDA, J. M. et al. Rede subterrânea residencial - RSR. Belo Horizonte: CEMIG, 2000. 11p. (Relatório DC/BS - 001/00)

BOCCUZZI, C. V. et al. Implantação de redes subterrâneas em condomínios residencial. Eletricidade Moderna, v. 25, n. 275, p. $90-100,1997$.

BROWNING, D. M. The economic impacts of deferring electric utility tree maintenance.

Arborist News, p.17-19, Apr. 1997.

COMPANHIAENERGÉTICADEMINAS GERAIS -

CEMIG. Viabilidade econômica de redes de distribuição protegidas. Belo Horizonte, 1998. 27p. (Relatório de Estudo de Distribuição, ED-3.40).

COMPANHIA PARANAENSE DE ENERGIA COPEL. Implantação de redes compactas no sistema de distribuição urbana de eletricidade. Paraná, 1995. 6p. (Programa SOS - Árvore).

PIRELLI. Fios e postes, desapareçam!. Revista Pirelli Club, n.12, p. 8-12. 2000.

SARDETO, E. Avaliação técnica, econômica e de impacto ambiental da implantação das redes compactas protegidas em Maringá, 1999. $71 \mathrm{f}$.

Monografia (Especialização) - Universidade Federal do Paraná, Curitiba, 1999.

SEITZ, R. A. Considerações sobre a poda de árvores na arborização urbana. In: ENCONTRO NACIONAL SOBREARBORIZAÇÃOURBANA, 3., Curitiba, 1990. Anais... Curitiba: FUPEF, 1990. p.87-100.

SOARES, M. P. Verdes urbanos e rurais: orientação para arborização de cidades e sítios campesinos. Porto Alegre: Cinco Continentes, 1998. 242p. 